\title{
Effect of flurbiprofen axetil combined with "Cocktail" therapy on opioid dosage in patients after total knee arthroplasty
}

\author{
Lu Wang', Li-xin Wu'2 Zhe Han \\ Wen-hai Ma ${ }^{4}$, Zhi-hui Geng ${ }^{5}$
}

\begin{abstract}
Objectives: To investigate the effect of flurbiprofen axetil combined with "cocktail" therapy on opioid dosage in patients after total knee arthroplasty (TKA).

Methods: The clinical data of 200 patients who underwent TKA in Baoding No.1 Central Hospital hospital from March 2019 to March 2021 were collected for retrospective analysis. All 200 patients were divided into two groups according to their intraoperative anesthesia methods: the control group (100 cases) and the experimental group (100 cases). Patients in the control group were treated with "cocktail" therapy intraoperatively, while those in the experimental group were treated with flurbiprofen axetil combined with "cocktail" therapy intraoperatively. The hip pain scores in resting state and motion state were compared between the two groups at different postoperative time points, and postoperative pain relief, adverse reactions, and patient satisfaction with analgesia were statistically analyzed to evaluate the postoperative quality of life of the patients.

Results: A statistically significant difference was observed in the intergroup and temporal effects of pain scores in resting state and motion state between the two groups $(p<0.05)$. By comparison at each time point, the pain scores in the experimental group were significantly lower than those in the control group at the time point T1-T6 in resting and motion states, with a statistically significant difference $(\mathrm{p}<0.05)$. The frequency and dosage of remedial medication per capita in the experimental group were significantly lower than those in the control group, with a statistical significance $(p<0.05)$. There was no significant difference in the scores of life quality items between the two groups preoperatively $(p>0.05)$, while the scores of each item in the experimental group were significantly higher than those in the control group postoperatively $(p<0.05)$. The satisfaction degree of the experimental group was significantly higher than that of the control group, showing a statistically significant difference $(p<0.05)$.

Conclusions: Flurbiprofen axetil combined with "cocktail" therapy is a safe treatment regimen that can improve the quality of life and safety of patients. With such a regimen, postoperative pain of patients undergoing TKA can be effectively relieved, and the use of opioids can be reduced.
\end{abstract}

KEYWORDS: “Cocktail” therapy, Flurbiprofen axetil, Opioids, Total knee arthroplasty.

doi: https://doi.org/10.12669/pjms.38.3.4735

How to cite this:

Wang L, Wu L, Han Z, Ma W, Geng Z. Effect of flurbiprofen axetil combined with "Cocktail" therapy on opioid dosage in patients after total knee arthroplasty. Pak J Med Sci. 2022;38(3):724-729. doi: https://doi.org/10.12669/pjms.38.3.4735

This is an Open Access article distributed under the terms of the Creative Commons Attribution License (http://creativecommons.org/licenses/by/3.0), which permits unrestricted use, distribution, and reproduction in any medium, provided the original work is properly cited.

Note: Lu Wang and Li-xin Wu are both considered as co-first authors.

Correspondence:

Zhi-hui Geng,

Department of Pharmacy,

Baoding No.1 Central Hospital,

Baoding 071000, Hebei, China.

E-mail: gengzhihui666@163.com

* Received for Publication:

* Revision Received:

* Revision Accepted: *
May 19, 2021

October 4, 2021

October 26, 2021

\section{INTRODUCTION}

Total knee arthroplasty (TKA), in orthopedic surgery, is a kind of surgery with large injury to the affected limb, which is characterized by relatively complicated surgery process, heavy bleeding, and severe postoperative pain. It has been shown in previous studies that the postoperative pain caused by TKA ranks the most severe among all surgical procedures, and nearly $1 / 5$ of patients are not satisfied 
with the postoperative analgesic effect. ${ }^{1}$ Currently, there is no unified clinical standard for postoperative analgesia of TKA. "Cocktail" therapy, namely nerve block and local injection of drugs around the joint, is a commonly studied postoperative analgesia method to date. ${ }^{2}$ Specifically, this therapy blocks pain transmission by applying drugs directly around the nerve. Nonetheless, shortcomings are inevitable: the short duration of the drug makes it difficult to achieve the purpose of long-term analgesia, and the pain may exacerbate after the failure of analgesic drugs. ${ }^{3}$ Opioids are often used for remedial analgesia postoperatively, but with more serious adverse reactions. In view of this, some researchers have proposed to combine "cocktail" therapy with other analgesic methods to prolong the analgesic effect. ${ }^{4}$ Flurbiprofen axetil, as a non-steroidal targeted analgesic drug, mainly inhibits the generation of coxidase via spinal cord and peripheral pathways and reduces prostaglandin synthesis, thereby increasing pain threshold in the body. ${ }^{5}$ Its advantage over other analgesics lies in its faster action effect, relatively long action time and targeted therapy.

Currently, few domestic studies on the combination of flurbiprofen axetil and "cocktail" therapy for analgesia have been reported. In this study, the safety and effectiveness of flurbiprofen axetil combined with "cocktail" therapy after TKA were investigated by indicators such as pain score, postoperative pain relief, and adverse reactions.

\section{METHODS}

The clinical data of 200 patients who underwent TKA in Baoding No.1 Central Hospital from March 2019 to March 2021 were collected for retrospective analysis.

\section{Inclusion criteria:}

- All patients receiving TKA treatment in our hospital due to knee osteoarthritis;
- Patients classified as grade II-III according to the American Society of Anesthesiologists (ASA);

- Patients with no history of using narcotic analgesics one week before going to bed;

- Patients without previous knee surgery;

- Patients with complete clinical data.

Ethical approval: The study was approved by the Institutional Ethics Committee of Baoding No.1 Central Hospital, (Date December 24, 2018) and written informed consent was obtained from all participants.

\section{Exclusion criteria:}

- Patients with mental disorders or language barriers;

- Patients with allergy or tolerance to the drug in this study;

- Patients with alcohol or drug dependence;

- Patients with a long history of opioid use;

- Patients with coagulation dysfunction, severe organ lesions and short term intolerance to surgery; - Patients with severe preoperative stiffness and deformation of the knee joint or severe instability caused by collateral ligament injury.

All patients were divided into two groups according to their intraoperative anesthesia methods: the control group (100 cases) and the experimental group (100 cases). There was no significant difference in general data between the two groups, which were comparable. Table-I.

Methods: Control group: The cocktail was prepared into a $100 \mathrm{ml}$ solution consisting of $200 \mathrm{mg}$ of ropivacaine injection (Astra Zeneca $\mathrm{AB}$, Import Drug Registration Certificate H20140763) + 5mg of morphine hydrochloride injection (Northeast Pharmaceutical Group Company Shenyang No.1 Pharmaceutical Co., Ltd., National Drug Approval No. H21022436) + normal saline. $50 \mathrm{ml}$ of the above cocktails were extracted and added into $40 \mathrm{mg}$ of triamcinolone acetonide injection (Kunming Jida

Table-I: Comparison of general data between the two groups.

\begin{tabular}{llcccc}
\hline General information & & Experimental group & Control group & $t / \chi^{2}$ & $p$ \\
\hline Gender (cases) & Male & 23 & 26 & 0.243 & 0.622 \\
Age (years old) & Female & 77 & 74 & & \\
Body mass index $\left(\mathrm{kg} / \mathrm{m}^{2}\right)$ & & $66.35 \pm 5.52$ & $66.22 \pm 5.93$ & 0.164 & 0.870 \\
Weight $(\mathrm{kg})$ & & $27.18 \pm 3.84$ & $27.62 \pm 3.92$ & 0.802 & 0.424 \\
& & $69.38 \pm 11.47$ & $67.27 \pm 11.43$ & 0.952 & 0.343 \\
ASA grading (cases) & Grade II & 62 & 67 & 0.546 & 0.460 \\
& Grade II & 38 & 33 & & \\
Affected knee position (cases) & Left knee & 54 & 52 & 0.080 & 0.777 \\
& Right knee & 46 & 48 & & \\
\hline
\end{tabular}


Pharmaceutical Co., Ltd., State Drug Approval No. H53021604). 50ml of the above cocktail was drawn and added $40 \mathrm{mg}$ of triamcinolone acetonide injection (Kunming Jida Pharmaceutical Co., Ltd., National Medicine Standard H53021604), with the injection site at the posterior joint capsule; $50 \mathrm{ml}$ cocktail without hormones was injected into MCL, LCL, quadriceps, subpatellar fat pad, other joint capsules and subcutaneous locations. Experimental group: 50mg flurbiprofen axetil injection (Beijing Tide Pharmaceutical Co., Ltd., State Drug Approval No. H20041508) was added into the above cocktails, and the other drug use and administration methods were the same as those in the control group. Remedial analgesia regimen: Patients were given dezocine (Yangtze River Pharmaceutical Group Co., Ltd., State Drug Approval No. H20080329) intramuscular injection in case of pain visual analogue scale (VAS) $\geq 4$ points.

Observation indicators:

Pain score: VAS was used to evaluate the pain degree of patients in both groups in the resting state and motion state at 6 time points of $6 \mathrm{~h}$ (T1), $12 \mathrm{~h}$ (T2), $24 \mathrm{~h}$ (T3), $48 \mathrm{~h}$ (T4), $72 \mathrm{~h}$ (T5) and $5 \mathrm{~d}$ (T6) postoperatively. 0 points indicates painless and 10 points means intolerable severe pain. The higher the score, the more severe the pain.

Postoperative pain relief: The number of postoperative pain relief and the dosage of remedial drugs per capita in the two groups were statistically analyzed. Analgesia-related adverse reactions: The incidence of postoperative adverse analgesic reactions, such as malignant vomiting and respiratory depression, was counted in the two groups.

Postoperative quality of life: The World Health Organization Quality of Life Scale (WHOQOL$\mathrm{BREF}^{6}$ was used to evaluate preoperative and postoperative quality of life, including the fields of physiology, psychology, social relations and environment. The postoperative quality of life of each patient was scored using a 1-5 scale, which was converted to a percentage scale. A higher score indicates a better quality of life.

Satisfaction: Patients' satisfaction was recorded during analgesia, and the satisfaction was divided into very satisfied (I), satisfied (II), fair (III) and poor (IV). Statistical Analysis: SPSS20.0 statistical software was used to analyze the data. Enumeration data were presented as $\mathrm{N}(\%), \chi 2$ or continuum-corrected $\chi 2$ test was performed, and rank sum test was used for grade data. Measurement data was expressed as $\bar{x} \pm s$, and t-test was performed. Multiple time points were compared by repeated measurement variance, and further pairwise comparison was performed by LSD-t test. $\mathrm{P}<0.05$ was considered statistically significant.

\section{RESULTS}

A statistically significant difference was observed in the inter-group and temporal effects of pain scores in resting state and motion state between the two groups $(p<0.05)$. By comparison at each time point, the pain scores in the experimental group were significantly lower than those in the control group at the time point T1-T6 in resting and motion states, with a statistically significant difference $(\mathrm{p}<0.05)$. Table-II.

The frequency and dosage of remedial medication per capita in the experimental group were significantly lower than those in the control group, with a statistical significance $(\mathrm{p}<0.05)$. Table-III. The incidence of nausea and vomiting in the experimental group was significantly lower than that in the control group $(p<0.05)$. No significant dif-

Table-II: Comparison of postoperative pain scores between the two groups ( $\bar{x} \pm s$, points).

\begin{tabular}{|c|c|c|c|c|c|c|c|}
\hline Group & & T1 & $T 2$ & T3 & T4 & T5 & T6 \\
\hline \multirow{5}{*}{$\begin{array}{l}\text { Resting } \\
\text { state }\end{array}$} & Experimental group & $1.26 \pm 0.38$ & $2.14 \pm 0.62$ & $2.18 \pm 0.67$ & $2.25 \pm 0.56$ & $2.07 \pm 0.43$ & $1.53 \pm 0.61$ \\
\hline & Control group & $1.38 \pm 0.41$ & $2.34 \pm 0.59$ & $2.65 \pm 0.63$ & $3.06 \pm 0.60$ & $3.18 \pm 0.51$ & $2.43 \pm 0.75$ \\
\hline & $\mathrm{F}_{\text {inter-group' }}, \mathrm{P}_{\text {inter-group }}$ & \multicolumn{6}{|c|}{$330.4, p<0.001$} \\
\hline & $\mathrm{F}_{\text {time' }^{\prime}} \mathrm{P}_{\text {time }}$ & \multicolumn{6}{|c|}{$153.12, \mathrm{p}<0.001$} \\
\hline & $\mathrm{F}_{\text {interaction' }}, \mathrm{P}_{\text {interaction }}$ & \multicolumn{6}{|c|}{$24.39, \mathrm{p}<0.001$} \\
\hline \multirow{5}{*}{$\begin{array}{l}\text { Motion } \\
\text { state }\end{array}$} & Experimental group & $1.54 \pm 0.53$ & $2.26 \pm 0.47$ & $2.37 \pm 0.54$ & $2.42 \pm 0.46$ & $2.38 \pm 0.44$ & $2.09 \pm 0.68$ \\
\hline & Control group & $1.85 \pm 0.61$ & $3.09 \pm 0.71$ & $3.49 \pm 0.58$ & $3.78 \pm 0.59$ & $4.05 \pm 0.56$ & $3.28 \pm 0.49$ \\
\hline & $\mathrm{F}_{\text {inter-group' }} \mathrm{P}_{\text {inter-group }}$ & \multicolumn{6}{|c|}{$33.35, p<0.001$} \\
\hline & $\mathrm{F}_{\text {time' }^{\prime}} \mathrm{P}_{\text {time }}$ & \multicolumn{6}{|c|}{$13.75, p<0.001$} \\
\hline & $\mathrm{F}_{\text {interaction' }}, \mathrm{P}_{\text {interaction }}$ & \multicolumn{6}{|c|}{$5.901, \mathrm{p}<0.001$} \\
\hline
\end{tabular}


Table-III: Comparison of postoperative pain relief between the two groups $(\bar{x} \pm s)$.

\begin{tabular}{lcc}
\hline Group & $\begin{array}{c}\text { No. of remedial } \\
\text { medication per } \\
\text { capita (times) }\end{array}$ & $\begin{array}{c}\text { Remediation } \\
\text { dose per } \\
\text { capita }(\mathrm{mg})\end{array}$ \\
\hline Experimental group & $0.93 \pm 0.29$ & $71.54 \pm 35.70$ \\
Control group & $1.07 \pm 0.45$ & $89.55 \pm 42.09$ \\
$\mathrm{t}$ & 2.615 & 3.025 \\
$\mathrm{p}$ & 0.010 & 0.003 \\
\hline
\end{tabular}

ference was observed in the incidence of other adverse reactions between the two groups $(p>0.05)$. Table-IV. There was no significant difference in the scores of life quality items between the two groups preoperatively $(p>0.05)$, while the scores of each item in the experimental group were significantly higher than those in the control group postoperatively $(\mathrm{p}<0.05)$. Table- $\mathrm{V}$. The satisfaction degree of the experimental group was significantly higher than that of the control group, showing a statistically significant difference $(\mathrm{p}<0.05)$. Table-VI.

\section{DISCUSSION}

TKA is considered to be an effective surgical procedure for the treatment of knee joint disease and osteoarthritis. However, it was found in Rice Da et al. ${ }^{7}$ study that $20 \%-70 \%$ of patients who received TKA had moderate to severe pain during the first 1-4 day. Failure to adequately control pain will not only lead to pathophysiological reactions, increase patient negative emotions, disrupt sleep, and reduce patient satisfaction postoperatively, but also increase the risk of complications such as deep vein thrombosis, wound infection, and delayed healing. ${ }^{8,9}$ Opioids, as first-line analgesics, boast a preferable analgesic effect, but the effect of dose control and postoperative pain treatment is difficult to be achieved due to its serious side effects. ${ }^{10}$ In view of this, a more effective analgesic regimen remains to be sought.

"Cocktail" therapy was first proposed by Australian scholars, which refers to the implementation of local infiltration analgesia by injecting local anesthetics, opioids or non-steroidal drugs into the posterior capsule of the knee joint and soft tissues around the surgical area by adding adrenaline or corticosteroids and other drugs. ${ }^{11}$ A previous study by Danoff Jr et al. ${ }^{12}$ showed that "cocktail" therapy used for TKA significantly reduced the severity of early acute pain and promoted early functional exercise of the knee joint. Moreover, a study by Chai $\mathrm{X}$ et $\mathrm{al}^{13}$ discussed many cases and also found that "cocktail" therapy can produce a preferable analgesic effect, with no significant increase in the incidence of adverse reactions. Flurbiprofen axetil is one of the clinically commonly used non-steroidal anti-inflammatory drugs (NSAIDs) in major orthopedic surgery. It has a high affinity for inflamed

Table-IV: Comparison of analgesia-related adverse reactions between the two groups [n(\%)].

\begin{tabular}{lccccc}
\hline Group & $\begin{array}{c}\text { Nausea and } \\
\text { vomiting }\end{array}$ & $\begin{array}{c}\text { Inadequate } \\
\text { analgesia }\end{array}$ & Erythra & $\begin{array}{c}\text { Respiratory } \\
\text { depression }\end{array}$ & Dizziness \\
\hline Experimental group & $3(3.00)$ & $2(2.00)$ & $2(2.00)$ & $1(1.00)$ & $4(4.00)$ \\
Control group & $10(10.00)$ & $6(6.00)$ & $5(5.00)$ & $3(3.00)$ & $9(9.00)$ \\
X2 or continuity correction & 4.031 & 1.172 & 0.592 & 0.255 & 2.057 \\
$\mathrm{p}$ & 0.045 & 0.279 & 0.442 & 0.614 & 0.152 \\
\hline
\end{tabular}

Table-V: Comparison of postoperative quality of life between the two groups ( $\bar{x} \pm s$, points).

\begin{tabular}{llcccc}
\hline Item & & Experimental group & Control group & $t$ & $p$ \\
\hline \multirow{2}{*}{ Physical field } & Before surgery & $56.79 \pm 5.32$ & $57.29 \pm 4.91$ & 0.691 & 0.491 \\
& After surgery & $65.75 \pm 6.18$ & $63.34 \pm 7.28$ & 2.524 & 0.012 \\
\multirow{2}{*}{ Psychological field } & Before surgery & $53.42 \pm 3.09$ & $53.98 \pm 2.86$ & 1.330 & 0.185 \\
& After surgery & $59.17 \pm 4.46$ & $57.15 \pm 5.37$ & 2.894 & 0.004 \\
\multirow{5}{*}{ Social relations field } & Before surgery & $55.71 \pm 4.28$ & $56.23 \pm 3.82$ & 0.906 & 0.366 \\
& After surgery & $62.75 \pm 3.25$ & $60.07 \pm 3.59$ & 5.534 & $<0.001$ \\
\multirow{2}{*}{ Environmental field } & Before surgery & $63.26 \pm 5.81$ & $62.93 \pm 5.43$ & 0.415 & 0.679 \\
& After surgery & $70.08 \pm 4.67$ & $68.74 \pm 3.95$ & 2.190 & 0.030 \\
\hline
\end{tabular}


Table-VI: Comparison of patient satisfaction between the two groups $[n(\%)]$.

\begin{tabular}{lcccc}
\hline Group & $I$ & $I I$ & $I I I$ & $I V$ \\
\hline Experimen- & 48 & 37 & 13 & $2(2.00)$ \\
tal group & $(48.00)$ & $(37.00)$ & $(13.00)$ & \\
Control & 37 & 35 & 20 & $8(8.00)$ \\
group & $(37.00)$ & $(35.00)$ & $(20.00)$ & \\
$\mathrm{Z}$ & \multicolumn{5}{c}{4.802} \\
$\mathrm{p}$ & \multicolumn{5}{c}{0.028} \\
\hline
\end{tabular}

tissues and can achieve targeted drug therapy and prolong the action time ${ }^{14}$ However, due to the limited analgesic effect of NSAIDs, flurbiprofen axetil is only an effective auxiliary analgesic of opioids for moderate to severe pain. It was shown in a study by Xiao $\mathrm{X}$ et al. ${ }^{15}$ that flurbiprofen axetil combined with opiates can have a stronger analgesic effect in patients with TKA. In this study, flurbiprofen axetil combined with "cocktail" therapy is used in the postoperative analgesia of patients with TKA. The results show that a statistically significant difference can be observed in the inter-group and temporal effects of pain scores in resting state and motion state between the two groups. By comparison at each time point, the pain scores in the experimental group are significantly lower than those in the control group at the time point T1-T6 in resting and motion states, with a statistically significant difference, and the frequency and dosage of remedial medication per capita in the experimental group are significantly lower than those in the control group, suggesting that the combination of the two analgesic regimens can more effectively relieve the acute pain after TKA and reduce the use of opioids compared to "cocktail" therapy alone. The reason may have a close bearing on the inhibition of inflammatory response at the surgical site and the alleviation of inflammatory pain. TKA, during the intraoperative surface repair of the knee joint, usually requires a large number of bone removal and release of surrounding soft tissues, which causes great trauma and stress response to the body, resulting in severe postoperative pain. Meanwhile, tourniquets need to be used intraoperatively to reduce bleeding and expand surgical field of vision, which will stimulate the body to release inflammatory factors and increase the degree of postoperative pain. ${ }^{6,17}$ In "cocktail" therapy, ropivacaine can inhibit sodium channels on nerve cells and block nerve excitatory conduction; As opioids, morphine can bind to the central and surrounding opioid receptors, inhibit the pain-sensing area of the cerebral cortex, and pro- duce strong analgesic effect. Triamcinolone acetonide is conducive to alleviating the stress response caused by local trauma and also relieving postoperative pain. ${ }^{18,19}$ Under the combined action of the above three drugs, the stimulation of the sensory nerve endings by the pain factors produced by local tissue damage can be blocked, thereby reducing the transmission of pain from the nerve endings to the central nervous system and achieving the purpose of controlling postoperative pain. In contrast, flurbiprofen axetil can inhibit cyclooxygenase to reduce arachidonic acid to produce prostaglandins, and at the same time inhibit the production of central prostaglandins, thereby reducing the sensitization of the central and peripheral nervous systems and achieving analgesic effects. ${ }^{20,21}$ It was shown in a study by Wang J et al. ${ }^{22}$ that flurbiprofen axetil can be targeted to accumulate on surgical incisions and inflammation sites, with rapid onset, and the blood concentration can reach the peak 5-10 minutes after intravenous administration. With the combination of two analgesic regimens, the analgesic effect can be synergistically exerted and the analgesic effect can be optimized.

Furthermore, the incidence of nausea and vomiting in the experimental group is significantly lower than that in the control group, and the quality of life score and satisfaction are significantly higher than those in the control group, suggesting that flurbiprofen axetil combined with "cocktail" therapy is safer and can improve the postoperative quality of life and satisfaction degree of analgesia in patients. To explain it, flurbiprofen axetil does not produce central side effects similar to morphine respiratory depression. It was confirmed by Wang $\mathrm{X}$ et al. ${ }^{23}$ that flurbiprofen axetil can not only reduce inflammatory reaction and induce neuroprotective effect, but also overcome the adverse effects of other COX inhibitors and reduce the occurrence of gastric mucosal adverse reactions. ${ }^{24}$ In addition, both flurbiprofen axil and "cocktail" therapy have been proved to have neither significant effect on the motor nerve of patients nor adverse effect on postoperative recovery of knee function in patients with TKA. With the improvement of postoperative analgesia, not only can the satisfaction of patients be improved, but also their quality of life can be improved by promoting early postoperative functional exercise. ${ }^{25}$

Limitations of the study: Nevertheless, deficiencies are still visible in this study: VAS is used to score the pain, and the results are subjective to a certain extent due to the different tolerance and expression 
of pain in different patients. At the same time, the short postoperative observation and follow-up time leads to the lack of comparison with long-term effects. In view of this, more rigorous experimental methods are expected to be designed in future studies to improve the above deficiencies.

\section{CONCLUSIONS}

Flurbiprofen axetil combined with "cocktail" therapy is a safe treatment regimen that can improve the quality of life and safety of patients. With such a regimen, postoperative pain of patients undergoing TKA can be effectively relieved, and the use of opioids can be reduced.

Funding: This study was sponsored by Medical Science Research Project of Hebei Province (20191238).

Declaration of conflicting interest: None.

\section{REFERENCES}

1. Momoli A, Giarretta S, Modena M, Micheloni GM. The painful knee after total knee arthroplasty: evaluation and management. Acta Biomed. 2017;88(2S):60-67. doi: 10.23750/abm.v88i2-S.6515

2. Teratani T. Effect of cocktail therapy after arthroscopic rotator cuff repair: a randomized, double-blind trial. J Shoulder Elbow Surg. 2020;29(7):1310-1315. doi: 10.1016/j.jse.2020.03.004

3. Barrington JW, Lovald ST, Ong KL, Watson HN, Emerson RH Jr. Postoperative Pain After Primary Total Knee Arthroplasty: Comparison of Local Injection Analgesic Cocktails and the Role of Demographic and Surgical Factors. J Arthroplasty. 2016;31(9-Suppl):288-292. doi: 10.1016/j.arth.2016.05.002

4. Kulkarni M, Mallesh M, Wakankar H, Prajapati R, Pandit H. Effect of Methylprednisolone in Periarticular Infiltration for Primary Total Knee Arthroplasty on Pain and Rehabilitation. J Arthroplasty. 2019;34(8):1646-1649. doi: 10.1016/j.arth.2019.04.060

5. Wang RD, Sheng XR, Guan WX, Wang M, Peng C, Yang YY, et al. Flurbiprofen axetil for postoperative analgesia in upper $a b$ dominal surgery: a randomized, parallel controlled, doubleblind, multicenter clinical study. Surg Today. 2020;50(7):749-756. doi: 10.1007/s00595-019-01951-1

6. Skevington SM, Lotfy M, O'Connell KA; WHOQOL Group. The World Health Organization's WHOQOL-BREF quality of life assessment: Psychometric properties and results of the international field trial. A report from the WHOQOL group. Qual Life Res. 2004;13(2):299-310. doi: 10.1023/B:QURE.0000018486.91360.00

7. Rice DA, Kluger MT, McNair PJ, Lewis GN, Somogyi AA, Borotkanics $R$, et al. Persistent postoperative pain after total knee arthroplasty: a prospective cohort study of potential risk factors. Br J Anaesth. 2018;121(4):804-812. doi: 10.1016/j.bja.2018.05.070

8. Rutherford RW, Jennings JM, Dennis DA. Enhancing Recovery After Total Knee Arthroplasty. Orthop Clin North Am. 2017;48(4):391-400. doi: 10.1016/j.ocl.2017.05.002

9. Sorel JC, Veltman ES, Honig A, Poolman RW. The influence of preoperative psychological distress on pain and function after total knee arthroplasty: a systematic review and meta-analysis. Bone Joint J. 2019;101-B(1):7-14. doi: 10.1302/0301-620X.101B1.BJJ-2018-0672.R1

10. Trasolini NA, McKnight BM, Dorr LD. The Opioid Crisis and the Orthopedic Surgeon. J Arthroplasty. 2018;33(11):3379-3382.e1. doi:10.1016/j.arth.2018.07.002.

11. Deng Z, Li Y, Storm GR, Kotian RN, Sun X, Lei G, et al. The efficiency and safety of steroid addition to multimodal cocktail periarticular injection in knee joint arthroplasty: a meta-analysis of randomized controlled trials. Sci Rep. 2019;9(1):7031. doi: 10.1038/s41598-019-43540-9

12. Danoff JR, Goel R, Henderson RA, Fraser J, Sharkey PF. Periarticular Ropivacaine Cocktail Is Equivalent to Liposomal Bupivacaine Cocktail in Bilateral Total Knee Arthroplasty. J Arthroplasty. 2018;33(8):2455-2459. doi: 10.1016/j.arth.2018.02.083
13. Chai X, Liu H, You C, Wang C. Efficacy of Additional Corticosteroid in a Multimodal Cocktail for Postoperative Analgesia Following Total Knee Arthroplasty: A Meta-Analysis of Randomized Controlled Trials. Pain Pract. 2019;19(3):316-327. doi: 10.1111/papr.12740

14. Wang K, Luo J, Zheng L, Luo T. Preoperative flurbiprofen axetil administration for acute postoperative pain: a meta-analysis of randomized controlled trials. J Anesth. 2017;31(6):852-860. doi: 10.1007/ s00540-017-2409-0

15. Xiao X, Zhang Q, Ouyang Z, Guo X. Comparison of perioperative flurbiprofen axetil or celecoxib administration for pain management after total-knee arthroplasty: A retrospective study. Medicine (Baltimore). 2018;97(37):e12391. doi: 10.1097/MD.0000000000012391

16. Lim HA, Song EK, Seon JK, Park KS, Shin YJ, Yang HY. Causes of Aseptic Persistent Pain after Total Knee Arthroplasty. Clin Orthop Surg. 2017;9(1):50-56. doi: 10.4055/cios.2017.9.1.50

17. Liu Y, Si H, Zeng Y, Li M, Xie H, Shen B. More pain and slower functional recovery when a tourniquet is used during total knee arthroplasty. Knee Surg Sports Traumatol Arthrosc. 2020;28(6):1842-1860. doi: 10.1007/s00167-019-05617-w

18. Iwakiri K, Ohta Y, Kobayashi A, Minoda Y, Nakamura H. Loca Efficacy of Periarticular Morphine Injection in Simultaneous Bilateral Total Knee Arthroplasty: A Prospective, Randomized, DoubleBlind Trial. J Arthroplasty. 2017;32(12):3637-3642. doi: 10.1016/j. arth.2017.07.020

19. Wang Y, Zhou A. A new improvement: subperiosteal cocktail application to effectively reduce pain and blood loss after total knee arthroplasty. J Orthop Surg Res. 2020;15(1):33. doi: 10.1186/s13018020-1563-5

20. Hao J, Wang K, Shao Y, Cheng X, Yan Z. Intravenous flurbiprofen axetil to relieve cancer-related multiple breakthrough pain: A clinical study. J Palliat Med. 2013;16(2):190-192. doi: 10.1089/jpm.2012.0353

21. Nonaka T, Hara M, Miyamoto C, Sugita M, Yamamoto T. Comparison of the analgesic effect of intravenous acetaminophen with that of flurbiprofen axetil on post-breast surgery pain: a randomized controlled trial. J Anesth. 2016;30(3):405-409. doi: 10.1007/s00540-016-2150-0

22. Wang J, Dong L, Wang R, Cai Y. Evaluation of bioequivalence of two flurbiprofen axetil injections: A randomized, open-label, double-cycle, and crossover study. Clin Exp Pharmacol Physiol. 2021;48(5):660 667. doi: 10.1111/1440-1681.13479

23. Wang X, Wang Y, Hu Y, Wang L, Zhao W, Wei L, et al. Effect of flurbiprofen axetil on postoperative delirium for elderly patients. Brain Behav. 2019;9(6):e01290. doi: 10.1002/brb3.1290

24. Xiang X, Yuan X, Lian Y, Fang J, Wu Y. Effect of oxycodone hydrochloride combined with flurbiprofen axetil for intravenous patient-controlled analgesia in lower abdominal patients: A randomized trial. Medicine (Baltimore). 2018;97(7):e9911. doi: 10.1097/ MD.0000000000009911

25. Canovas F, Dagneaux L. Quality of life after total knee arthroplasty Orthop Traumatol Surg Res. 2018;104(1S):S41-S46. doi: 10.1016/j. otsr.2017.04.017

Authors' Contributions: LW \& LXW: Designed this study and prepared this manuscript,and are responsible and accountable for the accuracy or integrity of the work. ZHhG: Collected and analyzed clinical data. ZH \& WHM: Were involved in the study and significantly revised this manuscript.

Authors:

1. Lu Wang

Department of Pharmacy,

2. Li-xin Wu,

Department of Pharmacy,

3. Zhe Han

Third Department of Orthopedics,

4. Wen-hai Ma,

Third Department of Orthopedics,

5. Zhi-hui Geng,

Department of Pharmacy,

1-5: Baoding No.1 Central Hospital,

Baoding 071000, Hebei, China. 